\title{
Digital Signal Modulation Recognition Equipment Based on High-order Cumulants
}

\author{
Han Bao ${ }^{1}$, Yongzhi Wang ${ }^{2}$, Lei Chen ${ }^{3}$ \\ 1. School of Software, University of Science and Technology LiaoNing, Anshan, LiaoNing, China \\ 2. College of Information and Communication Engineering, Harbin Engineering University, Harbin, China \\ Corresponding Author: Yongzhi Wang, 493366821@qq.com \\ 3. Department of Information Technology, College of Engineering and Computing, Georgia Southern University,

\begin{abstract}
For several reasons, modulation recognition is extremely important in wireless communication. In this paper, a common method, based on fourth-order and sixth-order cumulants, is proposed to recognize the modulation of digital signals in the device. Automatic modulation recognition is a fundamental step to perform the classification of different modulation formats. It is very important both in military and civil applications. Different signal transmission requires different modulation. These modulation makes user convenient to work in such communication field. This device is mainly used to recognize the modulation of the digital signals automatically. The function of the device is mainly used to distinguish different digital signals.
\end{abstract}

Keywords: automatic modulation recognition; wireless communication; digital signal

\section{Introduction}

Automatic classification of digital modulation is more and more important as the complexity of digital signal systems higher and higher and the number of the systems larger and larger. Modulation recognition of communication signals is often used for signal monitoring and spectrum management. Modulation recognition can also be used for electronic protection and attack in electronic support [1]. Besides, with the accelerated development of communication technology and the wide application of digital technology, the wireless communication environment is increasingly complex. Effective monitoring and identification of these communication signals is very important in military and civilian areas [2].

In this paper, the device can transmit and receive many kinds of modulation signals. Furthermore, it can recognize the modulation of the signals in the receiving end. The kinds of the modulation which the device can recognize are 16 quadrature amplitude modulation (16QAM), 8 phase-shift keying (8PSK), quadrature phase-shift keying (QPSK) and binary phase-shift keying (BPSK) [5]. And the algorithm based on the "I-Q image" is put forward in [6].

The recognition's method is a common, algorithm based on high-order cumulants (HOC) which can classify the above several modulations through threshold judgment [3]. This method can effectively classify phase-shift keying (PSK) and quadrature amplitude (QAM) modulation in-class. Thus, this algorithm can be used for simple identification. 


\section{High-order cumulants algorithm}

The r-order $\left(r=m_{1}+m_{2}+\cdots+m_{n}\right)$ mixed moments of a group of complex stationary random values $\left[a_{1}, a_{2}, \cdots, a_{n}\right]$ can be expressed as [4]

$$
\begin{aligned}
M\left[a_{1}^{m_{1}}, a_{2}^{m_{2}}, \cdots, a_{n}^{m_{n}}\right] & =E\left\{a_{1}^{m_{1}}, a_{2}^{m_{2}}, \cdots, a_{n}^{m_{n}}\right\} \\
& =(-i)^{r} \frac{\partial \Phi\left(\omega_{1}, \omega_{2}, \cdots, \omega_{n}\right)}{\partial \omega_{1}^{m_{1}} \partial \omega_{2}^{m_{2}} \cdots \partial \omega_{n}^{m_{n}}}
\end{aligned}
$$

Where the $\Phi\left(\omega_{1}, \omega_{2}, \cdots, \omega_{n}\right)=E\left\{\exp \left[j\left(\omega_{1} a_{1}+\omega_{2} a_{2}+\cdots \omega_{n} a_{n}\right)\right]\right\} \quad$ is the primary eigenfunction of the values, which is the mixed eigenfunction. And let the $\ln \Phi\left(\omega_{1}, \omega_{2}, \cdots \omega_{n}\right)$ be the next eigenfunction.

Well, for the groups of values $\left[a_{1}, a_{2}, \cdots, a_{n}\right]$, its r-order cumulants can be expressed as

$$
\operatorname{Cum}=\left[a_{1}^{m_{1}}, a_{2}^{m_{2}}, \cdots, a_{n}^{m_{n}}\right]=\left.(-j)^{r} \frac{\partial\left[\ln \Phi\left(\omega_{1}, \omega_{2}, \cdots, \omega_{n}\right)\right]}{\partial \omega_{1}^{m_{1}} \partial \omega_{2}^{m_{2}} \cdots \partial \omega_{n}^{m_{n}}}\right|_{\omega_{1}=\omega_{2}=\cdots=\omega_{n}=0}
$$

Then there is the connection between mixed moments and cumulants

$$
\begin{aligned}
& \text { Cum }=\left[a_{1}^{m_{1}}, a_{2}^{m_{2}}, \cdots, a_{n}^{m_{n}}\right] \\
& =\sum(-1)^{q-1}(q-1) ! E\left\{\prod_{i \in S_{1}} a_{i}\right\} E\left\{\prod_{i \in S_{2}} a_{i}\right\} \cdots E\left\{\prod_{i \in S_{q}} a_{i}\right\}
\end{aligned}
$$

The $\Sigma$ denotes the sum of the partitions $s_{1}, s_{2}, \cdots s_{q}$ when $q=1,2, \cdots, n$.

Specifically, we can define the two-order cumulants of $A(m)$ which is the zero-mean stationary random process as

$$
\begin{gathered}
C_{20}=E\left[A(m) A\left(m+l_{1}\right)\right] \\
C_{21}=E\left[A^{*}(m) A\left(m+l_{1}\right)\right]
\end{gathered}
$$

And the four-order cumulants of $A(m)$ can be expressed as

$$
\begin{aligned}
C_{40} & =C u m\left[A\left(m+l_{3}\right), A\left(m+l_{2}\right), A\left(m+l_{1}\right), A(m)\right] \\
& =E\left[A\left(m+l_{3}\right) A\left(m+l_{2}\right) A\left(m+l_{1}\right) A(m)\right] \\
& -E\left[A(m) A\left(m+l_{2}\right)\right] E\left[A\left(m+l_{1}\right) A\left(m+l_{3}\right)\right] \\
& -E\left[A(m) A\left(m+l_{1}\right)\right] E\left[A\left(m+l_{2}\right) A\left(m+l_{3}\right)\right] \\
& -E\left[A(m) A\left(m+l_{3}\right)\right] E\left[A\left(m+l_{1}\right) A\left(m+l_{2}\right)\right]
\end{aligned}
$$




$$
\begin{aligned}
C_{41} & =\operatorname{Cum}\left[A^{*}\left(m+l_{3}\right), A\left(m+l_{2}\right), A\left(m+l_{1}\right), A(m)\right] \\
& =E\left[A^{*}\left(m+l_{3}\right) A\left(m+l_{2}\right) A\left(m+l_{1}\right) A(m)\right] \\
& -E\left[A(m) A\left(m+l_{2}\right)\right] E\left[A\left(m+l_{1}\right) A^{*}\left(m+l_{3}\right)\right] \\
& -E\left[A(m) A\left(m+l_{1}\right)\right] E\left[A\left(m+l_{2}\right) A^{*}\left(m+l_{3}\right)\right] \\
& -E\left[A(m) A^{*}\left(m+l_{3}\right)\right] E\left[A\left(m+l_{1}\right) A\left(m+l_{2}\right)\right] \\
C_{42}= & C u m\left[A^{*}\left(m+l_{3}\right), A\left(m+l_{2}\right), A\left(m+l_{1}\right), A^{*}(m)\right] \\
= & E\left[A^{*}\left(m+l_{3}\right) A\left(m+l_{2}\right) A\left(m+l_{1}\right) A^{*}(m)\right] \\
- & E\left[A^{*}(m) A^{*}\left(m+l_{3}\right)\right] E\left[A\left(m+l_{1}\right) A\left(m+l_{2}\right)\right] \\
- & E\left[A^{*}(m) A\left(m+l_{2}\right)\right] E\left[A\left(m+l_{1}\right) A^{*}\left(m+l_{3}\right)\right] \\
- & E\left[A^{*}(m) A\left(m+l_{1}\right)\right] E\left[A\left(m+l_{2}\right) A^{*}\left(m+l_{3}\right)\right]
\end{aligned}
$$

Let $M_{p q}=E\left[A(m)^{p-q} A^{*}(m)^{q}\right]$, when $l_{1}=l_{2}=l_{3}=0$ the equations should be converted as follows

$$
\begin{gathered}
C_{20}=M_{20} \\
C_{21}=M_{21} \\
C_{40}=M_{40}-3 M_{20}^{2} \\
C_{41}=M_{41}-3 M_{21} M_{20} \\
C_{42}=M_{42}-\left|M_{20}\right|^{2}-2 M_{21}^{2}
\end{gathered}
$$

And

$$
C_{63}=M_{63}-6 M_{20} M_{41}-9 M_{42} M_{21}+18 M_{20}^{2} M_{21}+12 M_{21}^{3}
$$

We can estimate the corresponding sample as the above cumulants. We think of $X(k)$ as zeromean; in fact, the mean is token away before the estimation. The correlations of them are in the following

$$
\begin{gathered}
C_{20}=M_{20}=\frac{1}{N} \sum_{k=1}^{N} r_{k}^{2} \\
C_{21}=M_{21}=\frac{1}{N} \sum_{k=1}^{N}\left|r_{k}\right|^{2} \\
C_{40}=M_{40}-3 M_{20}^{2}=\frac{1}{N} \sum_{k=1}^{N} r_{k}^{4}-3 M_{20}^{2} \\
C_{41}=M_{42}-3 M_{21} M_{20}=\frac{1}{N} \sum_{k=1}^{N} r_{k}^{3} r_{k}^{*}-3 M_{21} M_{20}
\end{gathered}
$$




$$
\begin{gathered}
C_{42}=M_{42}-\left|M_{20}\right|^{2}-2 M_{21}^{2}=\frac{1}{N} \sum_{k=1}^{N}\left|r_{k}\right|^{4}-\left|M_{20}\right|^{2}-2 M_{21}^{2} \\
C_{63}=M_{63}-9 C_{42} C_{21}-6\left(C_{21}\right)^{3}=\frac{1}{N} \sum_{k=1}^{N}\left|r_{k}\right|^{6}-9 C_{42} C_{21}-6\left(C_{21}\right)^{3}
\end{gathered}
$$

Here, we think about the cumulative virtual value in (11)-(14). We derive the virtual value by averaging the no-noise signals. Table 1 shows the connection of the signal power and phase between the virtual and actual values.

Table 1. Theoretical values

\begin{tabular}{c|c|c|c|c}
\hline & $C_{40}$ & $C_{41}$ & $C_{42}$ & $C_{63}$ \\
\hline $2 \mathrm{ASK}$ & $-2 E^{2}$ & $-2 E^{2}$ & $-2 E^{2}$ & $16 E^{3}$ \\
\hline $4 \mathrm{ASK}$ & $-1.36 E^{2}$ & $-1.36 E^{2}$ & $-1.36 E^{2}$ & $8.32 E^{3}$ \\
\hline $2 \mathrm{PSK}$ & $2 E^{2}$ & $2 E^{2}$ & $2 E^{2}$ & $16 E^{3}$ \\
\hline $4 \mathrm{PSK}$ & $E^{2}$ & 0 & $E^{2}$ & $4 E^{3}$ \\
\hline 2FSK & 0 & 0 & $E^{2}$ & $4 E^{3}$ \\
\hline $4 \mathrm{FSK}$ & 0 & 0 & $E^{2}$ & $4 E^{3}$ \\
\hline $8 \mathrm{PSK}$ & 0 & 0 & $E^{2}$ & $4 E^{3}$ \\
\hline 16QAM & $0.68 E^{2}$ & 0 & $0.68 E^{2}$ & $2.08 E^{3}$ \\
\hline
\end{tabular}

In summary, we can design two parameters to identify the digital signals as follows

$$
\begin{aligned}
& f_{1}=C_{40} / C_{42} \\
& f_{2}=C_{41} / C_{42} \\
& f_{3}=C_{63}^{2} / C_{42}^{3}
\end{aligned}
$$

And based on the results from Table 1, we can divide the signal into five parts. The theoretical values of their parameters are as follows

$$
f_{r}=\left[f_{1}, f_{2}, f_{3}\right]=\left\{\begin{array}{cc}
{[1,1,32]} & 2 A S K / 2 P S K \\
{[1,0,16]} & 4 P S K \\
{[1,1,27.52]} & 4 A S K \\
{[0,0,16]} & 8 P S K / M F S K \\
{[1,0,13.76]} & 16 Q A M
\end{array}\right.
$$

The Figure 1, Figure 2 and Figure 3 show the various parameters of the digital signal simulation curve with SNR from 0 to $10 \mathrm{~dB}$. 


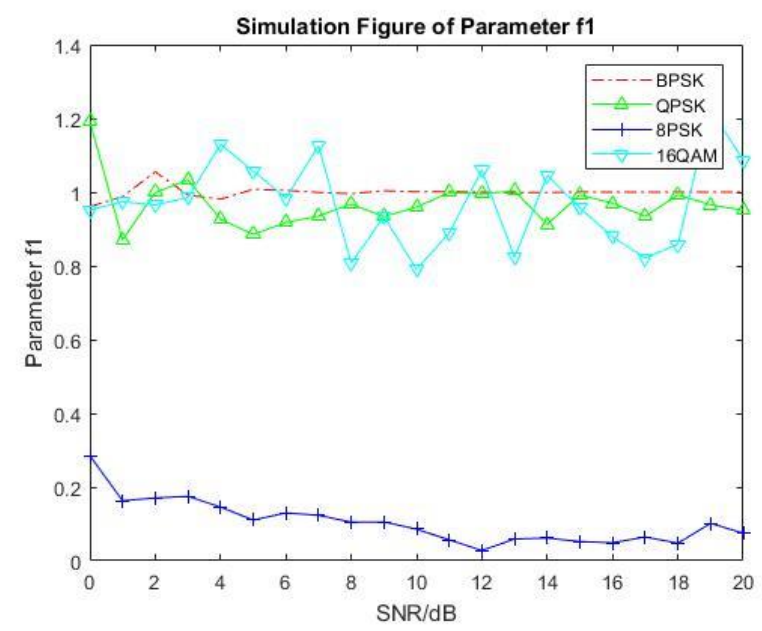

Fig. 1. The parameters of curve $f_{1}$ of various digital signals under different SNR

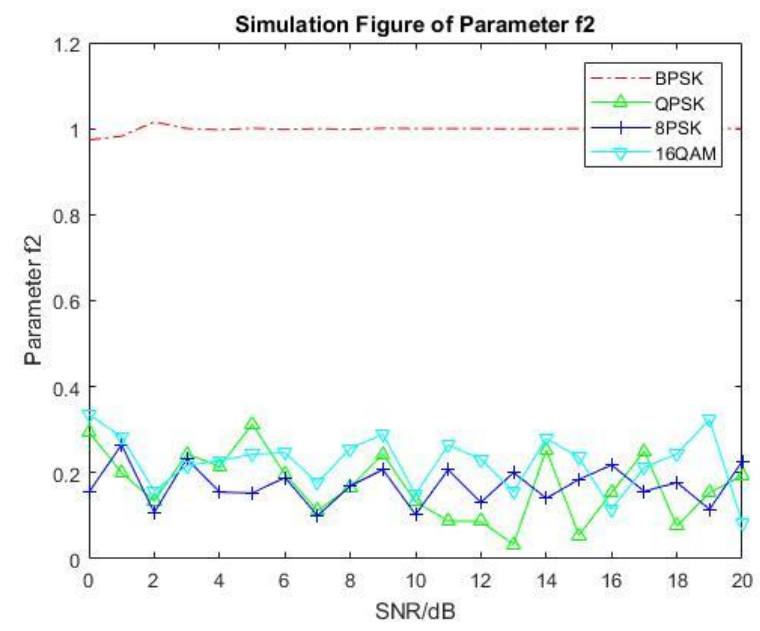

Fig. 2. The parameters of curve $f_{2}$ of various digital signals under different SNR

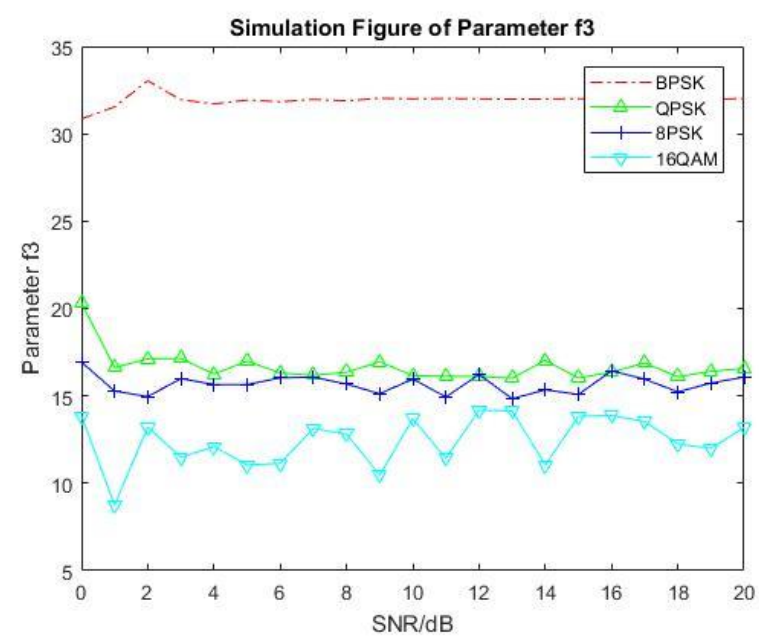

Fig. 3. The parameters of curve $f_{3}$ of various digital signals under different SNR 
Figure 1-3 show that the features of $f_{1} f_{2}, f_{3}$ can classify the four signals by setting threshold.

The feature's threshold of $f_{1}$ can be set to $0.5, f_{2}$ can be set to 0.7 and $f_{3}$ can be set to 14.5.

\section{Recognizing device}

The device is made of two parts which are transmitting part and receiving part. The former is used for transmitting digital signals while the latter is used for receiving signals and recognizing them. The program is based on LabVIEW and the communication device is USRP. The whole system is as Figure 4.

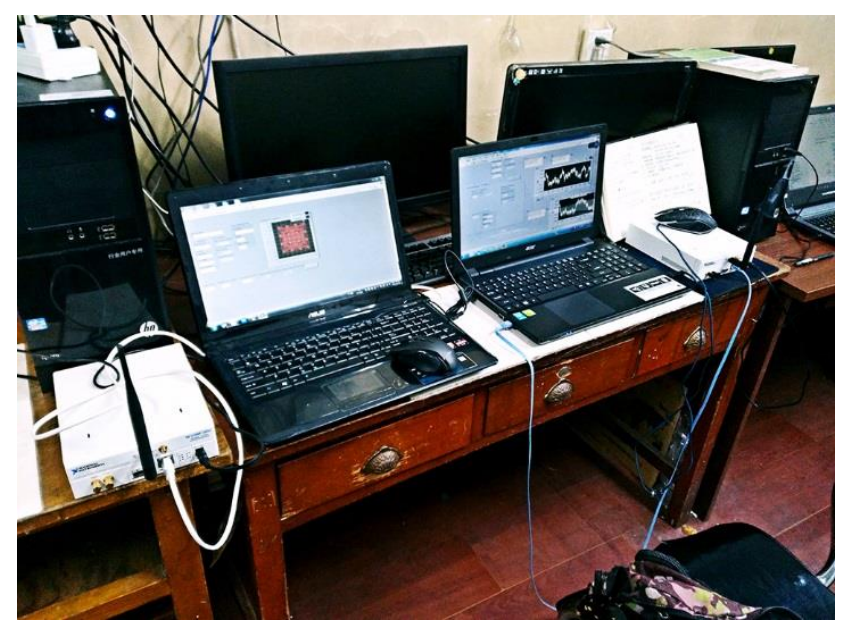

Fig. 4. The whole system

The device can classify BPSK, QPSK, 8PSK and 16QAM through the nature channel accurately. The following two pictures are the front panel of transmitting part and receiving part respectively. In transmitting part, we should set some parameters, and choose the modulation type to run the program. The display section has constellation graph and waveform. Then in receiving part, we should set some parameters to get the I/Q path spectrum, signal data and recognition result.

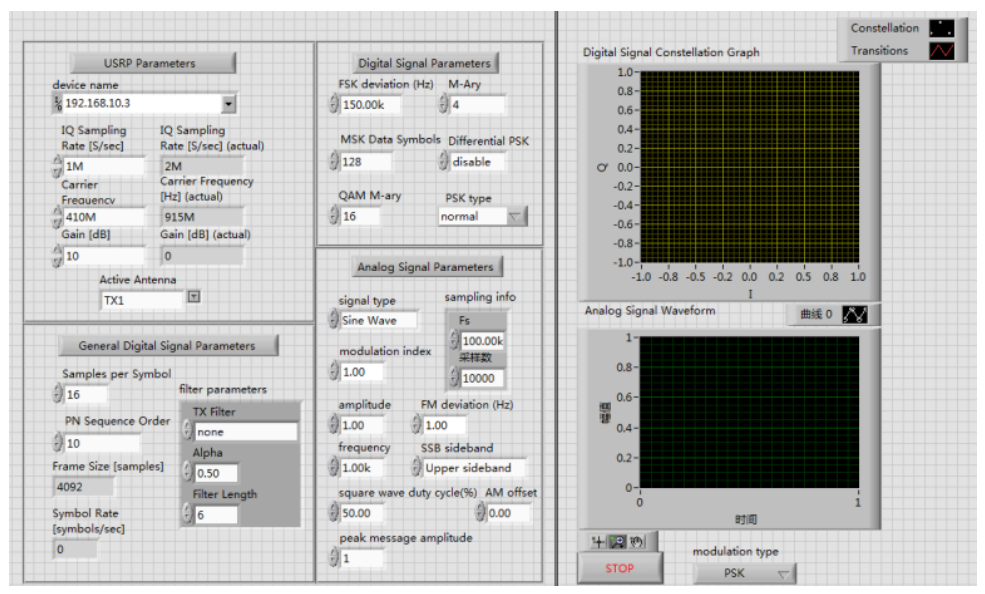

Fig. 5. The front panel of transmitting part 


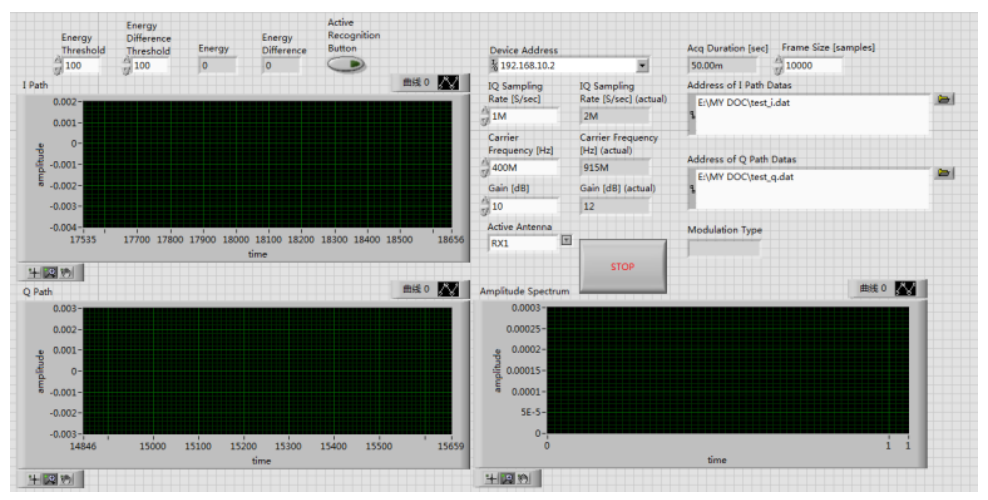

Fig. 6. The front panel of receiving part

For testing the recognition accuracy of the device, I collected 4 groups signals through the device. The 4 groups are BPSK, QPSK, 8PSK and 16QAM respectively. And there are 500000 points in each group. The carrier frequency of signals is $400 \mathrm{MHz}$, and the sampling rate of signals is $1 \mathrm{MHz}$. Then, I take the signals into the programs of MATLAB for computing the accuracy. I intercepted them to divide each group into 100 parts. To make it more straightforward, I made the result into a confusion matrix. The following figure is the confusion matrix of recognition result.

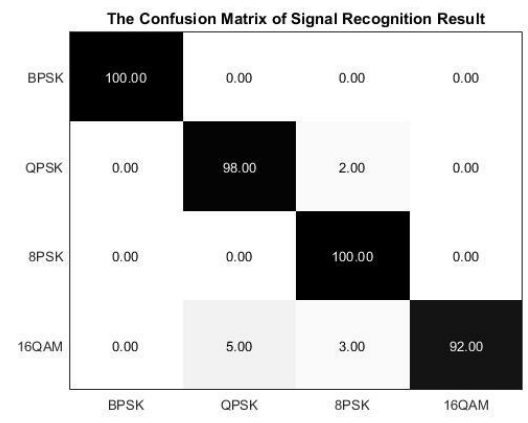

Fig. 7. The confusion matrix of recognition result

From the Figure 7. We can get $100 \%$ accuracy at BPSK and 8PSK. So their performance is almost great. Also, we can get 98\% accuracy at 8PSK, and 92\% accuracy at 16QAM. In that way, we think the accuracy of latter two signals is not bad, too.

\section{Conclusions}

In the article, the modulation recognition problem of the four signals was studied. The solution is to design a device based on LabVIEW which uses the high-order cumulants algorithm. The device allows us to perform real-time signal analysis. From the computing, we can conclude that the device can recognize 4 kinds of digitals with high accuracy which are BPSK, QPSK, 8PSK, 16QAM.

\section{References}

[1] Yu, H.; Guohua, W.; Chunyun, S.; Lanjian, L.: Hierarchical Digital Modulation Recognition Based on Higher-Order Cumulants, International Conference on Instrumentation \& Measurement, Computer, Communication and Control. 2012, 1645-1648. 
[2] Yong, Z.; Yitao, X.; Han, J.; Yijie, L.; Zhiwen, W.: Recognition of Digital Modulation Signals Based on High-order Cumulants. IEEE. 2015.

[3] Bhawna, Mukhwinder, K.; Lall, C, G.: Automatic Modulation Recognition for Digital Communication Signals. International Journal of Soft Computing and Engineering. 2012; Volume 2, pp. 2231-2307.

[4] Xinda, Z.: Automatic Communication Signal Modulation Base on Spectral Analysis. Radio Communication Technology Magn. China, 2003; Volume 2, pp. 30-33.

[5] Azzouz, E, E.; Nandi, K, A.: Automatic Modulation Recognition of Communication Signals. Norwell, MA: Kluwer, 1996.

[6] Hero, O, A.; Hadinejad-Mahram, H.: Digital Modulation Classification Using Power Moment Matrices. ICASSP. Seattle, WA, 1998; pp. 3285-3288. 